\title{
Article \\ Characteristics of Population Quality and Rice Quality of Semi-Waxy japonica Rice Varieties with Different Grain Yields
}

\author{
Qiuyuan Liu ${ }^{1,2}$, Shuang Chen ${ }^{2}$, Lei Zhou ${ }^{2}$, Yu Tao ${ }^{2}$, Jinyu Tian ${ }^{2}$, Zhipeng Xing ${ }^{2}{ }^{\oplus}$, Haiyan Wei ${ }^{2, *}$ \\ and Hongcheng Zhang ${ }^{2, *}$ \\ 1 Agricultural College, Xinyang Agriculture and Forestry University, Xinyang 464000, China; \\ liuqy@xyafu.edu.cn \\ 2 Jiangsu Key Laboratory of Crop Genetics and Physiology/Co-Innovation Center for Modern Production \\ Technology of Grain Crops, Yangzhou University, Yangzhou 225009, China; cs1692135738@163.com (S.C.); \\ zlzz1195@126.com (L.Z.); ty1067216721@163.com (Y.T.); dx120190081@yzu.edu.cn (J.T.); \\ zpxing@yzu.edu.cn (Z.X.) \\ * Correspondence: wei_haiyan@163.com (H.W.); hczhang@yzu.edu.cn (H.Z.)
}

check for updates

Citation: Liu, Q.; Chen, S.; Zhou, L.; Tao, Y.; Tian, J.; Xing, Z.; Wei, H.; Zhang, H. Characteristics of Population Quality and Rice Quality of Semi-Waxy japonica Rice Varieties with Different Grain Yields. Agriculture 2022, 12, 241. https:// doi.org/10.3390/agriculture 12020241

Academic Editor: Davinder Singh

Received: 29 December 2021

Accepted: 7 February 2022

Published: 8 February 2022

Publisher's Note: MDPI stays neutral with regard to jurisdictional claims in published maps and institutional affiliations.

Copyright: (C) 2022 by the authors. Licensee MDPI, Basel, Switzerland. This article is an open access article distributed under the terms and conditions of the Creative Commons Attribution (CC BY) license (https:// creativecommons.org/licenses/by/ $4.0 /)$.

\begin{abstract}
A primary focus of rice breeding and production is the optimization of yield and quality. Currently, semi-waxy japonica rice is widely planted in the middle and lower reaches of the Yangtze River due to its good eating quality and strong reputation among consumers. However, little information is yet available on grain yield formation and rice quality characteristics of these semiwaxy japonica rice varieties with different grain yields. In this study, three high-yielding (HGY) semi-waxy japonica rice varieties and three low-yielding (LGY) semi waxy japonica rice varieties were compared for population quality and rice quality in 2018 and 2019. The average values of spikelet per panicle, 1000-grain weight, and total spikelet number of the HGY varieties were significantly higher than those of the LGY varieties, while the panicle number and filled grain rate showed the opposite. Compared with the LGY varieties, the HGY varieties had a larger leaf area index at each growth stage, with a larger high efficient leaf area composed of a larger leaf length and width and smaller leaf angles of the top three leaves, as well as a greater single stem-sheath weight, more total dry matter accumulation, and longer growth duration from elongating to maturity. There were significant differences in rice quality between the HGY and LGY varieties. Compared with the LGY varieties, the head milled rice rate of the HGY varieties decreased significantly, and the chalky kernel rate and chalkiness degree increased significantly. Due to the low protein content, high peak viscosity, trough viscosity, and final viscosity and breakdown, as well as low setback, consistence, and pasting temperature of the HGY varieties, their taste values were significantly better than those of the LGY varieties. These results suggest that the HGY varieties could achieve a synergistic improvement of grain yield and eating quality, but the milling quality and appearance quality require further improvement.
\end{abstract}

Keywords: semi-waxy japonica rice; grain yield; rice quality; population quality

\section{Introduction}

As the global population increases, demand for rice is expected to double by 2050 [1]. To ensure food security, high grain yield has long been the primary goal of rice breeding and cultivation [2,3]. With socioeconomic development and continuous improvements in standards of living, the demand for good quality rice is rapidly increasing [4]. Therefore, the realization of high grain yield and good rice quality in rice will not only meet the growing market demand; but also increase economic benefits due to its good rice quality and high price.

Historically, a large number of studies have examined the mechanisms generating high-yield rice and required cultivation practices, as well as the characteristics of highyield rice varieties, such as greater total dry matter accumulation [5], larger sink capacity 
due to increased spikelet per panicle [6], higher leaf area and reduced decreasing rate of leaf area [7], improved canopy structure and root system [8], and more efficient use of temperature and light from elongating to maturity [9]. These findings have played a positive role in the breeding and cultivation of high-yield rice varieties, and the yield potential has significantly improved [10].

Rice quality traits dictate the market value and have a pivotal role in the adoption of new varieties. Rice quality traits include milling quality, appearance quality, nutritional quality, and cooking and eating quality. Previous studies have shown that rice quality traits are not only affected by cultivation techniques and the ecological environment, but there is also a close relationship with rice varieties [11-14]. Regarding the relationship between the grain yield and rice quality, some studies have indicated a deterioration in rice quality in the high-yield rice varieties [15], while other studies have also pointed out that grain yield and rice qualities, such as milling quality, appearance quality, and eating and cooking quality were significantly improved with the progress of breeding technology [16].

Semi-waxy japonica rice is a type of rice with an amylose content between $8 \%$ and $12 \%$ [17]. Due to the low amylose content being able to reduce the tendency of the starch granules to retrograde after cooling, cooked semi-waxy japonica rice is soft, elastic, and of moderate viscosity [18]. In recent years, these semi-waxy japonica varieties have attracted attention from breeders and rice physiologists, and as such, Nanjing 46 and Nanjing 9108 have been widely planted in the middle and lower reaches of the Yangtze River. Previous studies on semi-waxy japonica rice have mainly focused on the biological mechanisms driving its good eating quality [18] and the effects of related cultivation measures on its rice quality [11]. Similar to other types of rice, the grain yield of semi-waxy japonica rice showed significant differences across genotypes [12].

Until now, little information has been available on grain yield formation and the quality characteristics of these semi-waxy japonica rice varieties with different grain yields. It was hypothesized that the grain yield and rice quality of semi-waxy japonica rice varieties could be improved together. Therefore, we used semi-waxy japonica rice varieties with two different grain yield levels (with Nanjing5718, Nanjing9108, and Su1785 as the high-grainyield rice varieties, and Songzaoxiang NO.1, Changruan07-5, and Suxiangjing NO.3 as the low-grain-yield rice varieties) to study the population characteristics and the differences in rice quality in this experiment. The objective of this study was to (a) clarify the high-yield formation pathway of semi-waxy japonica rice varieties, and (b) evaluate the rice quality of semi-waxy japonica rice with a high grain yield. Such a study should provide useful information for achieving a high-quality and high-yield rice production system with inputs from both agronomy and breeding.

\section{Materials and Methods}

\subsection{Rice Varieties and Cultivation}

Six semi-waxy japonica rice varieties with two different grain yield levels (with Nanjing5718, Nanjing9108, and Su1785 as the high-grain-yield rice varieties, and Songzaoxiang NO.1, Changruan07-5, and Suxianging NO.3 as the low-grain-yield rice varieties) were used in this experiment. Field experiments were conducted during the rice-growing season in 2018 and repeated in 2019 at Shengao Town, Jiangyan District, Taizhou City, Jiangsu Province, China. The field soil is sandy soil, containing $15.73 \mathrm{~g} / \mathrm{kg}$ organic matter, $1.27 \mathrm{~g} / \mathrm{kg}$ total $\mathrm{N}, 76.83 \mathrm{mg} / \mathrm{kg}$ alkali hydrolyzable $\mathrm{N}, 16.11 \mathrm{mg} / \mathrm{kg}$ available $\mathrm{P}$, and $79.42 \mathrm{mg} / \mathrm{kg}$ available $\mathrm{K}$ in 2018. In 2019, the field soil contained $16.21 \mathrm{~g} / \mathrm{kg}$ organic matter, $1.45 \mathrm{~g} / \mathrm{kg}$ total $\mathrm{N}, 76.21 \mathrm{mg} / \mathrm{kg}$ alkali hydrolyzable $\mathrm{N}, 16.88 \mathrm{mg} / \mathrm{kg}$ available $\mathrm{P}$, and $85.41 \mathrm{mg} / \mathrm{kg}$ available $\mathrm{K}$.

In both years, all varieties (lines) were sown in seedbeds on 13 June and transplanted on 23 June into open fields with two seedlings per hill. The hill spacing was $25 \mathrm{~cm}$ row spacing with $6 \mathrm{~cm}$ plant spacing. The field experiments were arranged in a randomized block design with three replicates, and the size of each plot was $3 \mathrm{~m} \times 5 \mathrm{~m}$. Nutrient input included nitrogen $(\mathrm{N})$, phosphorus $(\mathrm{P})$, and potassium $(\mathrm{K})$ fertilizers. A total of $270 \mathrm{~kg} / \mathrm{ha}$ 
$\mathrm{N}$ was applied as urea $(46 \% \mathrm{~N}): 30 \%$ as basal fertilizer, $40 \%$ as tiller fertilizer, and $30 \%$ at the stage of panicle initiation. Urea was applied to all varieties at the same time. Calcium superphosphate $\left(\mathrm{P}_{2} \mathrm{O}_{5}\right.$ content: $\left.12 \%\right)$ was applied as a basal fertilizer at a rate of $135 \mathrm{~kg}$ $\mathrm{P}_{2} \mathrm{O}_{5} \mathrm{ha}^{-1}$. Similarly, potassium chloride $\left(\mathrm{K}_{2} \mathrm{O}\right.$ content: $\left.60 \%\right)$ was split into two equal amounts $\left(135 \mathrm{~kg} \mathrm{~K}_{2} \mathrm{O} \mathrm{ha}^{-1}\right)$ and applied around the emergence and booting stages. Field management followed established agronomic procedures.

\subsection{Sampling and Measurements}

\subsubsection{Dates of Elongating, Heading, and Maturity}

The date when the first internode length of more than $50 \%$ of the plants in the whole field reaches $2 \mathrm{~cm}$ is elongating stage. The period of more than $50 \%$ of the panicles extraction in the whole field is heading stage. Maturity refers to the date when more than $85 \%$ of the grains on a single panicle become hard and unbreakable, and more than $90 \%$ of the panicles in the whole field meet the above standards;

- $\quad$ Days before elongating $(\mathrm{d})=$ elongating date - sowing date;

- Days form elongating to heading $(\mathrm{d})=$ heading date - elongating date;

- Days form heading to maturity $(\mathrm{d})=$ maturity date - heading date;

- Days total growth duration $(\mathrm{d})=$ maturity date - sowing date.

\subsubsection{Dry Matter Weight and Leaf Area Index (LAI)}

Plants from three representative hills were uprooted from each plot at the elongating, heading, and maturity stages. Root portions were removed, and the remainder was separated into leaves, stems plus sheath, and panicles. LAI was measured with a portable leaf area meter (Li-3000A, LI-COR, Lincoln, Dearborn, MI, USA) at the elongating, heading, and maturity stages. The high effective leaf area (top 3 leaves area of effective tiller) was determined at the heading stage.

The high effective leaf area ratio $(\%)=($ the top 3 leaves area of the effective tiller at heading/total green leaf area at heading) $\times 100$.

Decreasing rate of leaf area $\left(\mathrm{LAI} \mathrm{d}^{-1}\right)=(\mathrm{LAI}$ at heading $-\mathrm{LAI}$ at maturity $) /$ days from heading to maturity.

Each component of the plants was bagged and oven-dried separately at $105{ }^{\circ} \mathrm{C}$ for $30 \mathrm{~min}$ and then at $80^{\circ} \mathrm{C}$ to a constant weight. Samples were then weighed to determine total dry matter weight.

\subsubsection{Top 3 Leaves Morphology and Single Stem-Sheath Weight}

At 10 days after heading, 15 representative single plants were randomly selected from each plot to determine leaf angle (the angle between leaf and stem) and length and width of the top 3 leaves. Then, all the stem-sheaths were bagged and oven-dried separately at $105^{\circ} \mathrm{C}$ for $30 \mathrm{~min}$ and then at $80^{\circ} \mathrm{C}$ to a constant weight. These stem-sheaths were then weighed to determine single stem-sheath weight.

\subsubsection{Grain Yield and Its Components}

At maturity, the number of panicles per $\mathrm{m}^{2}$ was determined from three representative square meter regions that were randomly sampled from each plot. Five plants with average panicle number were sampled randomly from each plot to determine the yield components, including spikelet per panicle, filled grain rate, and 1000-grain weight. Grain yield was determined from a harvest area of $5 \mathrm{~m}^{2}$ in the middle of each plot at maturity, and the grain yield was weighed. The final grain yield was adjusted to $14 \%$ moisture content.

- Harvest index = grain yield / total dry matter weight;

- Total spikelet number $=$ panicle number $\times$ spikelet per panicle. 


\subsubsection{Rice Quality}

A total of $1 \mathrm{~kg}$ of rice grains harvested from each tested variety was dried to a standard moisture content of $14 \%$ and stored for over 3 months. Characteristics of rice quality, including brown rice rate (BRR), milled rice rate (MRR), head milled rice rate (HMRR), chalky kernel rate (CKR), chalky area (CA), chalkiness degree (CD), and amylose content were measured according to GB/T17891-2017 [19]. To measure these traits, $100 \mathrm{~g}$ rice kernel samples were dehulled into brown rice by a laboratory dehuller (SY88-TH, BRIC, Korea), and the brown rice was processed into milled rice by a laboratory polisher (LTJM-2099, Hangzhou, China). After, the head milled rice was manually selected from the milled rice. A total of $10 \mathrm{~g}$ head milled rice was then scanned to analyze the CKR, CA, and CD by a rice appearance quality scanner (SC-E, Wanshen, Hangzhou, China). Amylose content was determined by iodine colorimetry at a wavelength of $620 \mathrm{~nm}$, using a potato starch standard mixture. Protein content was measured using a grain analyzer (Infratec 1241, Foss, Copenhagen, Denmark).

The rice flour pasting properties were determined using a Rapid Visco Analyser (RVA, Super3, Newport Scientific, Sydney, Australia), following the procedure of the American Association of Cereal Chemists. A total of $3 \mathrm{~g}$ of flour sifted with $0.15 \mathrm{~mm}$ sieves was mixed with $25 \mathrm{~g}$ deionized water in the RVA sample can. The peak viscosity, trough viscosity, final viscosity in $\mathrm{CP}$ (centipoise) units and their derivative parameters breakdown (peak viscosity-trough viscosity), setback (final viscosity-peak viscosity), and consistency (final viscosity-trough viscosity) were recorded with matching Software of Thermal Cline for Windows.

Eating quality was evaluated with a rice taste analyzer (STA1A, Satake Co., Hiroshima, Japan), which converted various physicochemical parameters of the rice into "taste value" scores (a comprehensive reflection of the rice's eating quality) based on correlations between the near-infrared reflectance measurements of the key constituents and preference sensory scores.

\subsection{Statistical Analysis}

Analysis of variance was performed using SPSS version 22. The sources of variation included year, grain yield type, rice variety, and the interactions of year $\times$ grain yield type and year $\times$ rice variety. Means were tested using the least significant difference (LSD) test at $p=0.05$. Tables were prepared using MS Excel 2013 for Windows.

\section{Results}

\subsection{Days of Different Growth Stages}

The total growth duration of the HGY varieties was 8 and 7 days longer than that of the LGY varieties in 2018 and 2019, respectively (Table 1). The duration from sowing to elongating stages and from heading to maturity stages was almost identical for the HGY and LGY varieties across both years, while the duration from elongating to heading stages of the HGY varieties was about 4 days longer than that of the LGY varieties.

\subsection{Grain Yield and Its Components}

There were significant genotypic differences in grain yield and its components across the HGY and LGY varieties (Table 2). Compared with the LGY varieties, the average grain yields of the HGY varieties were $29.39 \%$ higher in 2018 and $30.79 \%$ higher in 2019 . The spikelet per panicle and 1000-grain weight of the HGY varieties were significantly higher than those of the LGY varieties, but the panicle number and filled grain rate were reduced. The average total spikelet number of the HGY varieties was $18.42 \%$ higher in 2018 and $22.28 \%$ higher in 2019, compared to the LGY varieties, likely due to increased numbers of spikelet per panicle. 
Table 1. Dates of elongating, heading, and maturity of semi-waxy japonica rice varieties with different grain yields.

\begin{tabular}{|c|c|c|c|c|c|c|c|c|}
\hline Type & Variety & Elongating & Heading & Maturity & $\begin{array}{c}\text { Before } \\
\text { Elongating (d) }\end{array}$ & $\begin{array}{l}\text { Elongating to } \\
\text { Heading (d) }\end{array}$ & $\begin{array}{l}\text { Heading to } \\
\text { Maturity } \\
\text { (d) }\end{array}$ & $\begin{array}{l}\text { Total Growth } \\
\text { Duration } \\
\text { (d) }\end{array}$ \\
\hline \multicolumn{9}{|l|}{2018} \\
\hline \multirow{4}{*}{ HGY } & Nanjing5718 & 5 Aug & 2 Sep & $26 \mathrm{Oct}$ & 53 & 28 & 54 & 135 \\
\hline & Nanjing9108 & 5 Aug & 5 Sep & 29 Oct & 53 & 31 & 54 & 138 \\
\hline & Su1785 & 7 Aug & 5 Sep & 31 Oct & 55 & 29 & 56 & 140 \\
\hline & Mean & & & & 54 & 29 & 55 & 138 \\
\hline \multirow{4}{*}{ LGY } & $\begin{array}{c}\text { Songzaoxiang } \\
\text { NO. } 1\end{array}$ & 5 Aug & $28 \mathrm{Aug}$ & 20 Oct & 53 & 23 & 53 & 129 \\
\hline & Changruan07-5 & 4 Aug & 1 Sep & $23 \mathrm{Oct}$ & 52 & 28 & 52 & 129 \\
\hline & $\begin{array}{c}\text { Suxiangjing } \\
\text { NO.3 }\end{array}$ & 6 Aug & 30 Aug & $23 \mathrm{Oct}$ & 54 & 24 & 54 & 132 \\
\hline & Mean & & & & 53 & 25 & 53 & 130 \\
\hline 2019 & Nanjing5718 & 5 Aug & 2 Sep & 26 Oct & 53 & 28 & 54 & 135 \\
\hline \multirow[t]{3}{*}{ HGY } & Nanjing9108 & 5 Aug & 2 Sep & 26 Oct & 53 & 28 & 54 & 135 \\
\hline & Su1785 & 7 Aug & 2 Sep & $28 \mathrm{Oct}$ & 55 & 26 & 56 & 137 \\
\hline & Mean & & & & 54 & 27 & 55 & 136 \\
\hline \multirow{4}{*}{ LGY } & $\begin{array}{c}\text { Songzaoxiang } \\
\text { NO.1 }\end{array}$ & 5 Aug & 28 Aug & 20 Oct & 53 & 23 & 53 & 129 \\
\hline & Changruan07-5 & 5 Aug & 29 Aug & 20 Oct & 53 & 24 & 52 & 129 \\
\hline & $\begin{array}{c}\text { Suxiangjing } \\
\mathrm{NO}_{3}\end{array}$ & 5 Aug & 27 Aug & 20 Oct & 53 & 22 & 54 & 129 \\
\hline & Mean & & & & 53 & 23 & 53 & 129 \\
\hline
\end{tabular}

HGY, high-yield varieties; LGY, low-yield varieties.

Table 2. Grain yield and yield components of semi-waxy japonica rice varieties with different grain yields.

\begin{tabular}{|c|c|c|c|c|c|c|c|}
\hline Type & Variety & $\begin{array}{c}\text { Panicle } \\
\text { Number } \\
\left(\times 10^{4} \mathrm{ha}^{-1}\right)\end{array}$ & $\begin{array}{l}\text { Spikelet per } \\
\text { Panicle }\end{array}$ & $\begin{array}{c}\text { Total Spikelet } \\
\text { Number } \\
\left(\times 10^{6} \mathrm{ha}^{-1}\right)\end{array}$ & $\begin{array}{l}\text { Filled-Grain } \\
\text { Rate (\%) }\end{array}$ & $\begin{array}{l}\text { 1000-Grain } \\
\text { Weight } \\
\text { (g) }\end{array}$ & $\begin{array}{l}\text { Grain Yield } \\
\left(\mathrm{t} \mathrm{ha}^{-1}\right)\end{array}$ \\
\hline \multicolumn{8}{|l|}{2018} \\
\hline \multirow{5}{*}{ HGY } & Nanjing5718 & $286.46 \mathrm{f}$ & $123.33 \mathrm{a}$ & $353.31 \mathrm{~d}$ & $96.98 \mathrm{a}$ & $30.17 \mathrm{a}$ & $10.18 \mathrm{a}$ \\
\hline & Nanjing9108 & $345.23 \mathrm{~d}$ & $120.80 \mathrm{a}$ & $417.05 \mathrm{~b}$ & $91.13 \mathrm{~b}$ & $26.37 \mathrm{c}$ & $9.65 \mathrm{~b}$ \\
\hline & Su1785 & $386.83 \mathrm{~b}$ & $114.15 \mathrm{~b}$ & $441.56 \mathrm{a}$ & $88.93 \mathrm{~b}$ & $26.73 \mathrm{~b}$ & $10.02 \mathrm{ab}$ \\
\hline & Mean & 339.51 & 119.43 & 403.97 & 92.34 & 27.76 & 9.95 \\
\hline & $\begin{array}{c}\text { Songzaoxiang } \\
\text { NO.1 }\end{array}$ & $371.79 \mathrm{c}$ & $87.44 \mathrm{~d}$ & $325.10 \mathrm{e}$ & $94.82 \mathrm{a}$ & $25.93 \mathrm{~d}$ & $7.57 \mathrm{c}$ \\
\hline \multirow[t]{3}{*}{ LGY } & Changruan07-5 & $335.70 \mathrm{e}$ & $94.11 \mathrm{c}$ & $315.93 \mathrm{e}$ & $96.60 \mathrm{a}$ & $26.33 c$ & $7.91 \mathrm{c}$ \\
\hline & Suxiangjing NO.3 & $456.36 \mathrm{a}$ & $83.78 \mathrm{~d}$ & $382.34 \mathrm{c}$ & $96.22 \mathrm{a}$ & $20.93 \mathrm{e}$ & $7.58 \mathrm{c}$ \\
\hline & Mean & 387.95 & 88.44 & 341.12 & 95.88 & 24.40 & 7.69 \\
\hline \multicolumn{8}{|l|}{2019} \\
\hline \multirow{4}{*}{ HGY } & Nanjing9108 & $352.35 \mathrm{c}$ & $118.03 \mathrm{~b}$ & $415.87 \mathrm{a}$ & $90.88 \mathrm{~b}$ & $26.70 \mathrm{c}$ & $9.84 \mathrm{~b}$ \\
\hline & Su1785 & $373.05 \mathrm{~b}$ & $117.43 \mathrm{~b}$ & $438.07 \mathrm{a}$ & $86.20 \mathrm{c}$ & $27.07 \mathrm{~b}$ & $9.97 \mathrm{~b}$ \\
\hline & Mean & 340.30 & 120.62 & 409.16 & 91.82 & 28.04 & 10.11 \\
\hline & $\begin{array}{c}\text { Songzaoxiang } \\
\text { NO.1 }\end{array}$ & $343.80 \mathrm{c}$ & $92.89 \mathrm{~cd}$ & 319.35 c & $94.93 \mathrm{a}$ & $26.23 \mathrm{e}$ & $7.63 c$ \\
\hline \multirow[t]{3}{*}{ LGY } & Changruan07-5 & $327.08 \mathrm{~d}$ & $98.00 \mathrm{c}$ & $320.53 c$ & $96.93 \mathrm{a}$ & $26.60 \mathrm{~d}$ & $7.85 \mathrm{c}$ \\
\hline & Suxiangjing NO.3 & 433.88 a & $88.47 \mathrm{~d}$ & $363.96 \mathrm{~b}$ & $95.91 \mathrm{a}$ & $21.33 \mathrm{f}$ & $7.69 \mathrm{c}$ \\
\hline & Mean & 368.25 & 93.12 & 334.61 & 95.92 & 24.72 & 7.73 \\
\hline \multicolumn{8}{|c|}{ Analysis of variance } \\
\hline & Year (Y) & $* *$ & $*$ & ns & ns & $* *$ & ns \\
\hline & Type (T) & $* *$ & $* *$ & $* *$ & $* *$ & $* *$ & $* *$ \\
\hline & ariety (V) & $* *$ & $* *$ & $* *$ & $* *$ & $* *$ & $* *$ \\
\hline & $\mathrm{Y} \times \mathrm{T}$ & $* *$ & ns & ns & ns & ns & ns \\
\hline & $\mathrm{Y} \times \mathrm{V}$ & $*$ & ns & ns & ns & $* *$ & ns \\
\hline
\end{tabular}

HGY, high-yield varieties; LGY, low-yield varieties. Values within the same year followed by different letters are significantly different at the 0.05 probability level. * significant at the 0.05 probability level, ${ }^{* *}$ significant at the 0.01 probability level, ns not significant at the 0.05 probability level. 


\subsection{Dry Matter Accumulation and Harvest Index}

Compared with the LGY varieties, the dry matter accumulation of the HGY varieties was higher at each stage (Table 3). Before elongating, there was no significant difference in dry matter accumulation, but the dry matter accumulation ratio of the LGY varieties was significantly higher than that of HGY varieties in both years. During the period from elongating to heading, the average dry matter accumulation of the HGY varieties was $25.90 \%$ higher in 2018 and $28.95 \%$ higher in 2019 than that of the LGY varieties, while there was no significant difference in the dry matter accumulation ratio between the HGY and LGY varieties. At maturity, the total dry matter accumulation of the HGY varieties was more than $20 \mathrm{tha}^{-1}$, and was $27.79 \%$ higher in 2018 and $29.04 \%$ higher in 2019 than that of the LGY varieties, and the dry matter accumulation ratio of the HGY varieties was significantly higher than that of the LGY varieties. There was no significant difference in the harvest index among the six tested varieties, while the average harvest index of the HGY varieties was significantly higher than that of the LGY varieties.

Table 3. Dry matter accumulation and harvest index of semi-waxy japonica rice varieties with different grain yields.

\begin{tabular}{|c|c|c|c|c|c|c|c|c|c|}
\hline \multirow[b]{2}{*}{ Type } & \multirow[b]{2}{*}{ Variety } & \multicolumn{2}{|c|}{ Before Elongating } & \multicolumn{2}{|c|}{ Elongating to Heading } & \multicolumn{2}{|c|}{ Heading to Maturity } & \multirow{2}{*}{$\begin{array}{l}\text { Total Dry Matter } \\
\text { Accumulation } \\
\left(\mathrm{t} \mathrm{ha} \mathbf{a}^{-1}\right)\end{array}$} & \multirow{2}{*}{$\begin{array}{c}\text { Harvest } \\
\text { Index }\end{array}$} \\
\hline & & $\begin{array}{l}\text { Amount } \\
\left(\mathrm{t} \mathrm{ha}^{-1}\right)\end{array}$ & $\begin{array}{c}\text { Ratio } \\
(\%)\end{array}$ & $\begin{array}{c}\text { Amount } \\
\left(\mathrm{t} \mathrm{ha} \mathrm{a}^{-1}\right)\end{array}$ & Ratio (\%) & $\begin{array}{l}\text { Amount } \\
\left(\mathrm{t} \mathrm{ha}^{-1}\right)\end{array}$ & Ratio (\%) & & \\
\hline \multicolumn{10}{|l|}{2018} \\
\hline \multirow{5}{*}{ HGY } & Nanjing5718 & $3.32 \mathrm{a}$ & $15.64 b$ & $8.86 \mathrm{a}$ & $41.76 \mathrm{a}$ & $9.05 \mathrm{a}$ & $42.61 \mathrm{a}$ & $21.23 \mathrm{a}$ & $0.480 \mathrm{a}$ \\
\hline & Nanjing9108 & $3.35 \mathrm{a}$ & $16.53 \mathrm{~b}$ & $8.44 \mathrm{a}$ & $41.64 \mathrm{a}$ & $8.47 \mathrm{a}$ & $41.83 \mathrm{a}$ & $20.26 \mathrm{~b}$ & $0.476 \mathrm{a}$ \\
\hline & Su1785 & $3.34 \mathrm{a}$ & $16.14 b$ & $8.79 \mathrm{a}$ & $42.40 \mathrm{a}$ & $8.60 \mathrm{a}$ & $41.46 \mathrm{a}$ & $20.74 \mathrm{ab}$ & $0.483 \mathrm{a}$ \\
\hline & Mean & 3.34 & 16.10 & 8.70 & 41.93 & 8.71 & 41.97 & 20.74 & 0.480 \\
\hline & $\begin{array}{c}\text { Songzaoxiang } \\
\text { NO.1 }\end{array}$ & $3.18 \mathrm{a}$ & $19.39 \mathrm{a}$ & $6.94 \mathrm{~b}$ & $41.73 \mathrm{a}$ & $6.37 \mathrm{~b}$ & $38.88 \mathrm{~b}$ & $16.38 \mathrm{~cd}$ & $0.463 \mathrm{a}$ \\
\hline \multirow[t]{3}{*}{ LGY } & Changruan07-5 & $3.17 \mathrm{a}$ & $19.00 \mathrm{a}$ & $7.16 \mathrm{~b}$ & $42.84 \mathrm{a}$ & $6.38 \mathrm{~b}$ & $38.16 \mathrm{~b}$ & $16.71 \mathrm{c}$ & $0.474 \mathrm{a}$ \\
\hline & $\begin{array}{c}\text { Suxiangjing } \\
\text { NO.3 }\end{array}$ & $3.11 \mathrm{a}$ & $19.56 \mathrm{a}$ & $6.63 \mathrm{~b}$ & $41.73 \mathrm{a}$ & $6.21 \mathrm{~b}$ & $39.09 \mathrm{~b}$ & $15.88 \mathrm{~d}$ & 0.478 a \\
\hline & Mean & 3.10 & 19.31 & 6.91 & 41.97 & 6.32 & 38.71 & 16.32 & 0.471 \\
\hline \multirow{5}{*}{ HGY } & Nanjing5718 & $3.39 \mathrm{a}$ & $15.80 \mathrm{~b}$ & $9.05 \mathrm{a}$ & $42.15 \mathrm{a}$ & $9.03 \mathrm{a}$ & $42.05 \mathrm{a}$ & $21.48 \mathrm{a}$ & $0.489 \mathrm{a}$ \\
\hline & Nanjing9108 & $3.19 \mathrm{a}$ & $15.65 \mathrm{~b}$ & $8.72 \mathrm{a}$ & $42.72 \mathrm{a}$ & $8.48 \mathrm{a}$ & $41.55 \mathrm{a}$ & $20.40 \mathrm{~b}$ & $0.483 \mathrm{a}$ \\
\hline & Su1785 & $3.35 \mathrm{a}$ & $16.45 \mathrm{~b}$ & $8.42 \mathrm{a}$ & $41.66 \mathrm{a}$ & $8.61 \mathrm{a}$ & $42.23 \mathrm{a}$ & $20.38 \mathrm{~b}$ & $0.489 \mathrm{a}$ \\
\hline & Mean & 3.31 & 15.97 & 8.73 & 42.09 & 8.70 & 41.94 & 20.75 & 0.487 \\
\hline & $\begin{array}{c}\text { Songzaoxiang } \\
\text { NO.1 }\end{array}$ & $3.19 \mathrm{a}$ & $20.00 \mathrm{a}$ & $6.67 \mathrm{~b}$ & $41.85 \mathrm{a}$ & $6.08 \mathrm{~b}$ & $38.15 \mathrm{~b}$ & $15.93 \mathrm{~cd}$ & $0.479 \mathrm{a}$ \\
\hline \multirow[t]{3}{*}{ LGY } & Changruan07-5 & $3.17 \mathrm{a}$ & $19.04 \mathrm{a}$ & $7.12 \mathrm{~b}$ & $42.72 \mathrm{a}$ & $6.37 \mathrm{~b}$ & $38.24 b$ & $16.66 \mathrm{c}$ & $0.471 \mathrm{a}$ \\
\hline & $\begin{array}{l}\text { Suxiangjing } \\
\text { NO.3 }\end{array}$ & $3.14 \mathrm{a}$ & $20.07 \mathrm{a}$ & $6.52 \mathrm{~b}$ & $41.66 \mathrm{a}$ & $5.99 \mathrm{~b}$ & $38.27 \mathrm{~b}$ & $15.65 \mathrm{~d}$ & $0.492 \mathrm{a}$ \\
\hline & Mean & 3.17 & 19.70 & 6.77 & 42.07 & 6.15 & 38.22 & 16.08 & 0.481 \\
\hline \multicolumn{10}{|c|}{ Analysis of variance } \\
\hline & Year (Y) & ns & ns & ns & ns & ns & ns & ns & ns \\
\hline & Type (T) & $* *$ & $* *$ & $* *$ & ns & $* *$ & $* *$ & $* *$ & $*$ \\
\hline & Jariety (V) & ns & * & $* *$ & ns & $*$ & ns & $* *$ & ns \\
\hline & $\mathrm{Y} \times \mathrm{T}$ & ns & * & ns & ns & ns & ns & ns & ns \\
\hline & $\mathrm{Y} \times \mathrm{V}$ & ns & ns & ns & ns & ns & ns & ns & ns \\
\hline
\end{tabular}

HGY, high-yield varieties; LGY, low-yield varieties. Values within the same year followed by different letters are significantly different at the 0.05 probability level. ${ }^{*}$ significant at the 0.05 probability level, ${ }^{* *}$ significant at the 0.01 probability level, ns not significant at the 0.05 probability level.

\subsection{Leaf Area Index (LAI)}

The HGY varieties had increased LAI across different stages and years (Table 4). The average LAI of the HGY varieties was $25.89,17.28$, and $51.50 \%$ higher than that of the LGY varieties at the elongating, heading, and maturity stages, respectively, in 2018, and $20.72,17.86$, and $52.87 \%$ higher at those stages in 2019. The high effective LAI and high effective LAI ratio of the HGY varieties were both significantly higher than those of the LGY varieties in both years. The decreasing rate of leaf area of the HGY varieties was significantly lower than that of the LGY varieties in both years. 
Table 4. LAI at the main growth stages of semi-waxy japonica rice varieties with different grain yields.

\begin{tabular}{|c|c|c|c|c|c|c|c|}
\hline \multirow[b]{2}{*}{ Type } & \multirow[b]{2}{*}{ Variety } & \multirow[b]{2}{*}{ Elongating } & \multicolumn{3}{|c|}{ Heading } & \multirow[b]{2}{*}{ Maturity } & \multirow{2}{*}{$\begin{array}{l}\text { Decreasing Rate } \\
\text { of Leaf Area } \\
\text { (LAI d }{ }^{-1} \text { ) }\end{array}$} \\
\hline & & & Total LAI & $\begin{array}{l}\text { High Effective } \\
\text { LAI }\end{array}$ & $\begin{array}{l}\text { High Effective } \\
\text { LAI Ratio (\%) }\end{array}$ & & \\
\hline \multicolumn{8}{|l|}{2018} \\
\hline \multirow{5}{*}{ HGY } & Nanjing5718 & $4.93 \mathrm{a}$ & $8.21 \mathrm{a}$ & $5.43 \mathrm{a}$ & $66.14 \mathrm{a}$ & $4.24 \mathrm{a}$ & $0.074 \mathrm{ab}$ \\
\hline & Nanjing9108 & $4.37 \mathrm{bc}$ & $7.64 \mathrm{~b}$ & $5.19 \mathrm{~b}$ & $67.87 \mathrm{a}$ & $3.83 \mathrm{~b}$ & $0.071 \mathrm{~b}$ \\
\hline & Su1785 & $4.55 \mathrm{~b}$ & $8.18 \mathrm{a}$ & $5.50 \mathrm{a}$ & $67.19 \mathrm{a}$ & $4.01 \mathrm{ab}$ & $0.074 \mathrm{ab}$ \\
\hline & Mean & 4.62 & 8.01 & 5.37 & 67.07 & 4.03 & 0.073 \\
\hline & $\begin{array}{c}\text { Songzaoxiang } \\
\text { NO.1 }\end{array}$ & $3.34 \mathrm{~d}$ & $6.82 \mathrm{c}$ & $4.36 \mathrm{~cd}$ & $63.92 \mathrm{~b}$ & $2.66 \mathrm{~cd}$ & $0.078 \mathrm{ab}$ \\
\hline \multirow[t]{3}{*}{ LGY } & Changruan07-5 & $4.11 \mathrm{c}$ & $6.99 c$ & $4.50 \mathrm{c}$ & $64.39 \mathrm{~b}$ & $2.94 \mathrm{c}$ & $0.078 \mathrm{ab}$ \\
\hline & Suxiangjing NO.3 & $3.55 \mathrm{~d}$ & $6.69 c$ & $4.21 \mathrm{~d}$ & $63.03 \mathrm{~b}$ & $2.39 \mathrm{~d}$ & $0.080 \mathrm{a}$ \\
\hline & Mean & 3.67 & 6.83 & 4.36 & 63.78 & 2.66 & 0.079 \\
\hline \multicolumn{7}{|l|}{2019} & $0.075 \mathrm{ab}$ \\
\hline \multirow{4}{*}{ HGY } & Nanjing9108 & $4.13 \mathrm{ab}$ & $7.85 \mathrm{~b}$ & $5.28 \mathrm{~b}$ & $67.28 \mathrm{a}$ & $3.90 \mathrm{~b}$ & $0.073 \mathrm{~b}$ \\
\hline & Su1785 & $4.43 \mathrm{ab}$ & $7.99 \mathrm{~b}$ & $5.29 \mathrm{~b}$ & $66.26 \mathrm{a}$ & $3.79 \mathrm{~b}$ & $0.075 \mathrm{ab}$ \\
\hline & Mean & 4.37 & 8.05 & 5.40 & 66.99 & 3.99 & 0.074 \\
\hline & $\begin{array}{c}\text { Songzaoxiang } \\
\text { NO.1 }\end{array}$ & $3.37 \mathrm{c}$ & $6.90 \mathrm{c}$ & $4.38 \mathrm{c}$ & $63.51 \mathrm{~b}$ & $2.75 \mathrm{c}$ & $0.078 \mathrm{ab}$ \\
\hline \multirow[t]{3}{*}{ LGY } & Changruan07-5 & $3.99 \mathrm{~b}$ & $6.86 c$ & $4.39 \mathrm{c}$ & $64.09 \mathrm{~b}$ & $2.77 \mathrm{c}$ & $0.079 \mathrm{ab}$ \\
\hline & Suxiangjing NO.3 & $3.48 \mathrm{c}$ & $6.73 c$ & $4.33 \mathrm{c}$ & $64.30 \mathrm{~b}$ & $2.31 \mathrm{~d}$ & $0.082 \mathrm{a}$ \\
\hline & Mean & 3.62 & 6.83 & 4.37 & 63.97 & 2.61 & 0.080 \\
\hline \multicolumn{8}{|c|}{ Analysis of variance } \\
\hline & Year $(Y)$ & * & ns & ns & $\mathrm{ns}$ & ns & $\mathrm{ns}$ \\
\hline & Type (T) & $* *$ & $* *$ & $* *$ & $* *$ & $* *$ & ** \\
\hline & ariety (V) & $* *$ & $* *$ & $* *$ & $* *$ & $* *$ & $\mathrm{~ns}$ \\
\hline & $\mathrm{Y} \times \mathrm{T}$ & ns & ns & ns & ns & ns & $\mathrm{ns}$ \\
\hline & $\mathrm{Y} \times \mathrm{V}$ & ns & ns & $*$ & $* *$ & ns & ns \\
\hline
\end{tabular}

HGY, high-yield varieties; LGY, low-yield varieties. Values within the same year followed by different letters are significantly different at the 0.05 probability level. * significant at the 0.05 probability level, ${ }^{* *}$ significant at the 0.01 probability level, ns not significant at the 0.05 probability level.

\subsection{Morphology of the Top Three Leaves and Single Stem-Sheath Weight}

There were significant differences in the morphology of the top 3 leaves and single stem-sheath weight between the HGY and LGY varieties (Table 5). The single stem-sheath weight of the HGY varieties was significantly higher than that of the LGY varieties in both years, and the average single stem-sheath weight of the HGY varieties was $37.86 \%$ higher than that of the LGY varieties in 2018, and 38.19\% higher in 2019. The average length and width of the top three leaves of the HGY varieties were significantly higher than those of the LGY varieties, while the leaf angle of the top 3 leaves was reduced.

\subsection{Rice Quality}

3.6.1. Milling Quality and Appearance Quality

Some metrics of milling quality and appearance quality varied across the varieties (Table 6). There was no detectable difference in brown rice rate (BRR) or milled rice rate (MRR) between the different grain yield types. The head milled rice rate (HMRR) of the LGY varieties was $7.04 \%$ higher than that of the HGY varieties in 2018 , and $17.60 \%$ higher in 2019. Significant differences in appearance quality between the HGY and LGY varieties were observed: the average chalky kernel rate (CKR), chalky area (CA), and chalkiness degree (CD) of the HGY varieties were significantly higher than those of the LGY varieties.

\subsubsection{Pasting Properties}

There were significant differences in pasting properties among the varieties (Table 7). The average values of peak viscosity, trough viscosity, and final viscosity and breakdown of the HGY varieties were all significantly higher than those of the LGY varieties, while the average values of setback, consistence, and pasting temperature were all significantly lower than those of the LGY varieties. 
Table 5. Single stem-sheath weight and morphology of the top three leaves of semi-waxy japonica rice varieties with different grain yields.

\begin{tabular}{|c|c|c|c|c|c|c|c|c|c|c|c|}
\hline \multirow[b]{2}{*}{ Type } & \multirow[b]{2}{*}{ Variety } & \multirow{2}{*}{$\begin{array}{c}\text { Single } \\
\text { Stem-Sheath } \\
\text { Weight } \\
\text { (g) }\end{array}$} & \multicolumn{3}{|c|}{ Flag Leaf } & \multicolumn{3}{|c|}{ 2nd Leaf } & \multicolumn{3}{|c|}{ 3nd Leaf } \\
\hline & & & $\begin{array}{c}\text { Length } \\
(\mathrm{cm})\end{array}$ & $\begin{array}{c}\text { Width } \\
(\mathrm{cm})\end{array}$ & $\begin{array}{c}\text { Leaf Angles } \\
\left({ }^{\circ}\right)\end{array}$ & $\begin{array}{l}\text { Length } \\
\text { (cm) }\end{array}$ & $\begin{array}{l}\text { Width } \\
(\mathrm{cm})\end{array}$ & $\begin{array}{c}\text { Leaf Angles } \\
\left({ }^{\circ}\right)\end{array}$ & $\begin{array}{c}\text { Length } \\
\text { (cm) }\end{array}$ & $\begin{array}{c}\text { Width } \\
(\mathrm{cm})\end{array}$ & $\begin{array}{c}\text { Leaf Angles } \\
\left({ }^{\circ}\right)\end{array}$ \\
\hline \multicolumn{12}{|l|}{2018} \\
\hline \multirow{5}{*}{ HGY } & Nanjing5718 & $2.31 \mathrm{a}$ & $29.18 \mathrm{a}$ & $2.22 \mathrm{a}$ & $10.36 \mathrm{c}$ & $39.50 \mathrm{a}$ & $1.68 \mathrm{a}$ & $17.06 \mathrm{c}$ & $39.54 \mathrm{a}$ & $1.40 \mathrm{a}$ & $22.44 \mathrm{~b}$ \\
\hline & Nanjing9108 & $1.77 \mathrm{~b}$ & $27.12 \mathrm{a}$ & $1.74 \mathrm{c}$ & $10.46 \mathrm{c}$ & $38.96 \mathrm{a}$ & $1.42 \mathrm{c}$ & $16.84 \mathrm{c}$ & $34.62 \mathrm{~b}$ & $1.24 \mathrm{~b}$ & $23.02 \mathrm{~b}$ \\
\hline & Su1785 & $1.71 \mathrm{~b}$ & $27.60 \mathrm{a}$ & $1.90 \mathrm{~b}$ & $10.44 \mathrm{c}$ & $36.68 \mathrm{ab}$ & $1.54 \mathrm{~b}$ & $16.50 \mathrm{c}$ & $35.82 \mathrm{~b}$ & $1.44 \mathrm{a}$ & $23.24 \mathrm{~b}$ \\
\hline & Mean & 1.93 & 27.97 & 1.95 & 10.42 & 38.38 & 1.55 & 16.80 & 36.66 & 1.36 & 22.90 \\
\hline & $\begin{array}{c}\text { Songzaoxiang } \\
\text { NO.1 }\end{array}$ & $1.45 \mathrm{~d}$ & $30.34 \mathrm{a}$ & $1.64 \mathrm{c}$ & $14.18 \mathrm{a}$ & $34.84 \mathrm{ab}$ & $1.30 \mathrm{~d}$ & $21.36 \mathrm{~b}$ & $31.40 \mathrm{~b}$ & $1.20 \mathrm{~b}$ & $26.42 \mathrm{a}$ \\
\hline \multirow[t]{3}{*}{ LGY } & Changruan07-5 & $1.62 \mathrm{c}$ & $24.48 \mathrm{a}$ & $1.60 \mathrm{c}$ & $12.74 \mathrm{~b}$ & $34.20 \mathrm{ab}$ & $1.44 \mathrm{c}$ & $21.20 \mathrm{~b}$ & $31.90 \mathrm{~b}$ & $1.22 \mathrm{~b}$ & $26.06 \mathrm{a}$ \\
\hline & $\begin{array}{c}\text { Suxiangjing } \\
\text { NO.3 }\end{array}$ & $1.13 \mathrm{e}$ & $24.48 \mathrm{a}$ & $1.40 \mathrm{~d}$ & $14.14 \mathrm{a}$ & $31.94 \mathrm{~b}$ & $1.14 \mathrm{e}$ & $22.04 \mathrm{a}$ & $31.64 \mathrm{~b}$ & $1.04 \mathrm{c}$ & $26.00 \mathrm{a}$ \\
\hline & Mean & 1.40 & 26.43 & 1.55 & 13.69 & 33.66 & 1.29 & 21.53 & 31.65 & 1.15 & 26.16 \\
\hline \multirow{5}{*}{ HGY } & Nanjing5718 & $2.38 \mathrm{a}$ & $29.92 \mathrm{a}$ & $2.18 \mathrm{a}$ & $10.30 \mathrm{c}$ & $38.00 \mathrm{a}$ & $1.78 \mathrm{a}$ & $16.56 \mathrm{c}$ & $36.82 \mathrm{a}$ & $1.50 \mathrm{a}$ & $22.80 \mathrm{~b}$ \\
\hline & Nanjing9108 & $1.83 \mathrm{~b}$ & $27.30 \mathrm{a}$ & $1.72 \mathrm{c}$ & $10.44 \mathrm{c}$ & $35.46 \mathrm{a}$ & $1.44 \mathrm{bc}$ & $16.84 \mathrm{c}$ & $34.96 \mathrm{a}$ & $1.32 \mathrm{~b}$ & $23.16 \mathrm{~b}$ \\
\hline & Su1785 & $1.77 \mathrm{~b}$ & $26.78 \mathrm{a}$ & $1.88 \mathrm{~b}$ & $9.86 \mathrm{c}$ & $37.06 \mathrm{a}$ & $1.52 \mathrm{~b}$ & $17.28 \mathrm{c}$ & $36.54 \mathrm{a}$ & $1.42 \mathrm{a}$ & $23.34 \mathrm{~b}$ \\
\hline & Mean & 1.99 & 28.00 & 1.93 & 10.20 & 36.84 & 1.58 & 16.89 & 36.11 & 1.41 & 23.10 \\
\hline & $\begin{array}{c}\text { Songzaoxiang } \\
\text { NO.1 }\end{array}$ & $1.51 \mathrm{~d}$ & $28.62 \mathrm{a}$ & $1.58 \mathrm{c}$ & $13.96 \mathrm{a}$ & $31.64 \mathrm{~b}$ & $1.24 \mathrm{de}$ & $20.82 \mathrm{~b}$ & $31.84 \mathrm{~b}$ & $1.10 \mathrm{c}$ & $25.72 \mathrm{a}$ \\
\hline \multirow[t]{3}{*}{ LGY } & Changruan07-5 & $1.63 c$ & $21.82 \mathrm{a}$ & $1.58 \mathrm{c}$ & $12.12 \mathrm{~b}$ & $30.06 \mathrm{~b}$ & $1.36 \mathrm{~cd}$ & $20.90 \mathrm{~b}$ & $31.58 \mathrm{~b}$ & $1.26 \mathrm{~b}$ & $25.26 \mathrm{a}$ \\
\hline & $\begin{array}{c}\text { Suxiangjing } \\
\text { NO.3 }\end{array}$ & $1.17 \mathrm{e}$ & $22.56 \mathrm{a}$ & $1.38 \mathrm{~d}$ & $14.12 \mathrm{a}$ & $28.08 \mathrm{~b}$ & $1.14 \mathrm{e}$ & $21.66 \mathrm{a}$ & $29.08 \mathrm{~b}$ & $1.04 \mathrm{c}$ & $25.54 \mathrm{a}$ \\
\hline & Mean & 1.44 & 24.33 & 1.51 & 13.40 & 29.93 & 1.25 & 21.13 & 30.83 & 1.13 & 25.51 \\
\hline \multicolumn{12}{|c|}{ Analysis of variance } \\
\hline & Year (Y) & $* *$ & ns & ns & * & $* *$ & ns & ns & ns & ns & ns \\
\hline & Type (T) & $* *$ & $*$ & $* *$ & $* *$ & $* *$ & $* *$ & $* *$ & $* *$ & $* *$ & $* *$ \\
\hline & Variety (V) & $* *$ & $* *$ & $* *$ & $* *$ & ns & $* *$ & $* *$ & * & $* *$ & * \\
\hline & $\mathrm{Y} \times \mathrm{T}$ & ns & ns & ns & ns & ns & ns & ns & ns & ns & $* *$ \\
\hline & $\mathrm{Y} \times \mathrm{V}$ & ns & ns & ns & ns & ns & ns & ns & ns & ns & ns \\
\hline
\end{tabular}

HGY, high-yield varieties; LGY, low-yield varieties. Values within the same year followed by different letters are significantly different at the 0.05 probability level. * significant at the 0.05 probability level, ${ }^{* *}$ significant at the 0.01 probability level, ns not significant at the 0.05 probability level.

Table 6. Milling quality and appearance quality of semi-waxy japonica rice varieties with different grain yields.

\begin{tabular}{|c|c|c|c|c|c|c|c|}
\hline \multirow{2}{*}{ Type } & \multirow{2}{*}{ Variety } & \multicolumn{3}{|c|}{ Milling Quality (\%) } & \multicolumn{3}{|c|}{ Appearance Quality (\%) } \\
\hline & & BRR & MRR & HMRR & CKR & CA & CD \\
\hline \multicolumn{8}{|l|}{2018} \\
\hline \multirow{4}{*}{ HGY } & Nanjing5718 & $84.97 \mathrm{a}$ & $71.41 \mathrm{a}$ & $56.91 \mathrm{ab}$ & $35.50 \mathrm{a}$ & $27.68 \mathrm{a}$ & $9.82 \mathrm{a}$ \\
\hline & Nanjing9108 & $85.25 \mathrm{a}$ & $72.56 \mathrm{a}$ & $57.56 \mathrm{ab}$ & $33.01 \mathrm{a}$ & $23.98 \mathrm{c}$ & $7.92 \mathrm{~b}$ \\
\hline & Su1785 & 83.53 a & $72.43 \mathrm{a}$ & $54.30 \mathrm{~b}$ & $36.93 \mathrm{a}$ & $25.29 \mathrm{abc}$ & $9.34 \mathrm{a}$ \\
\hline & Mean & 84.59 & 72.13 & 56.26 & 35.14 & 25.65 & 9.03 \\
\hline \multirow{4}{*}{ LGY } & Songzaoxiang NO.1 & $83.73 \mathrm{a}$ & $72.99 \mathrm{a}$ & $60.29 \mathrm{a}$ & $25.98 \mathrm{~b}$ & $22.85 \mathrm{c}$ & $5.94 \mathrm{c}$ \\
\hline & Changruan07-5 & $85.79 \mathrm{a}$ & $72.18 \mathrm{a}$ & $59.35 \mathrm{a}$ & $24.87 \mathrm{~b}$ & $24.64 \mathrm{bc}$ & $6.11 \mathrm{c}$ \\
\hline & Suxiangjing NO.3 & $85.54 \mathrm{a}$ & $73.59 \mathrm{a}$ & $61.03 \mathrm{a}$ & $23.42 \mathrm{~b}$ & $27.14 \mathrm{ab}$ & $6.35 c$ \\
\hline & Mean & 85.02 & 72.92 & 60.22 & 24.75 & 24.88 & 6.13 \\
\hline \multirow{3}{*}{ HGY } & Nanjing5718 & $84.21 \mathrm{a}$ & $72.24 \mathrm{a}$ & $56.59 \mathrm{c}$ & $35.71 \mathrm{a}$ & $30.92 \mathrm{a}$ & $11.04 \mathrm{a}$ \\
\hline & Nanjing9108 & $85.11 \mathrm{a}$ & $73.16 \mathrm{a}$ & $53.27 \mathrm{~d}$ & $32.86 \mathrm{~b}$ & $29.90 \mathrm{a}$ & $9.81 \mathrm{~b}$ \\
\hline & Su1785 & $83.29 \mathrm{a}$ & $72.08 \mathrm{a}$ & $52.21 \mathrm{~d}$ & $30.53 \mathrm{~b}$ & $30.23 \mathrm{a}$ & $9.22 \mathrm{~b}$ \\
\hline \multirow{5}{*}{ LGY } & Mean & 84.20 & 72.49 & 54.03 & 33.03 & 30.35 & 10.02 \\
\hline & Songzaoxiang NO.1 & $83.43 \mathrm{a}$ & $74.30 \mathrm{a}$ & $66.76 \mathrm{a}$ & $23.81 \mathrm{c}$ & $22.19 \mathrm{~b}$ & $5.28 \mathrm{~d}$ \\
\hline & Changruan07-5 & $85.04 \mathrm{a}$ & $72.37 \mathrm{a}$ & $59.39 \mathrm{~b}$ & $23.23 \mathrm{c}$ & $23.89 \mathrm{~b}$ & $5.55 \mathrm{~d}$ \\
\hline & Suxiangjing NO.3 & $84.11 \mathrm{a}$ & $74.82 \mathrm{a}$ & $64.48 \mathrm{a}$ & $23.15 \mathrm{c}$ & $28.49 \mathrm{a}$ & $6.60 \mathrm{c}$ \\
\hline & Mean & 84.19 & 73.83 & 63.54 & 23.40 & 24.86 & 5.81 \\
\hline \multicolumn{8}{|c|}{ Analysis of variance } \\
\hline & Year $(Y)$ & ns & ns & ns & $* *$ & $* *$ & * \\
\hline & Type (T) & ns & ns & $* *$ & $* *$ & $* *$ & $* *$ \\
\hline & Jariety (V) & ns & ns & $* *$ & ns & $* *$ & $* *$ \\
\hline & $\mathrm{Y} \times \mathrm{T}$ & ns & ns & $* *$ & ns & $* *$ & $* *$ \\
\hline & $\mathrm{Y} \times \mathrm{V}$ & ns & ns & $* *$ & $*$ & ns & $* *$ \\
\hline
\end{tabular}

HGY, high-yield varieties; LGY, low-yield varieties. BRR, brown rice rate; MRR, milled rice rate; HMRR, head milled rice rate. CKR, chalky kernel rate; $C A$, chalky area; $C D$, chalkiness degree. Values within the same year followed by different letters are significantly different at the 0.05 probability level. * significant at the 0.05 probability level, ${ }^{* *}$ significant at the 0.01 probability level, ns not significant at the 0.05 probability level. 
Table 7. Pasting properties of semi-waxy japonica rice varieties with different grain yields.

\begin{tabular}{|c|c|c|c|c|c|c|c|c|}
\hline Type & Variety & $\begin{array}{l}\text { Peak Viscosity } \\
\text { (cP) }\end{array}$ & $\begin{array}{c}\text { Trough } \\
\text { Viscosity (cP) }\end{array}$ & $\begin{array}{c}\text { Final } \\
\text { Viscosity (cP) }\end{array}$ & $\begin{array}{l}\text { BREAKDOWN } \\
\text { (cP) }\end{array}$ & Setback (cP) & $\begin{array}{l}\text { Consistence } \\
\text { (cP) }\end{array}$ & $\begin{array}{c}\text { Pasting } \\
\text { Temperature } \\
\left({ }^{\circ} \mathrm{C}\right)\end{array}$ \\
\hline \multicolumn{9}{|l|}{2018} \\
\hline \multirow{4}{*}{ HGY } & Nanjing5718 & 3149 a & $1620 \mathrm{a}$ & 2118 a & 1529 a & $-1031 \mathrm{c}$ & $499 \mathrm{c}$ & $72.38 \mathrm{a}$ \\
\hline & Nanjing9108 & $2612 \mathrm{~d}$ & 1406 a & 1987 a & $1206 \mathrm{~b}$ & $-625 a b$ & $581 \mathrm{~b}$ & $71.68 \mathrm{a}$ \\
\hline & Su1785 & $2733 \mathrm{~b}$ & 1443 a & 1959 a & $1290 \mathrm{~b}$ & $-774 \mathrm{~b}$ & $516 \mathrm{c}$ & $71.28 \mathrm{a}$ \\
\hline & Mean & 2831 & 1490 & 2021 & 1341 & -810 & 532 & 71.78 \\
\hline \multirow{4}{*}{ LGY } & Songzaoxiang NO.1 & $2682 c$ & $1334 \mathrm{a}$ & $1900 \mathrm{a}$ & $1348 \mathrm{~b}$ & $-782 \mathrm{~b}$ & $566 \mathrm{~b}$ & $72.00 \mathrm{a}$ \\
\hline & Changruan07-5 & $2740 \mathrm{~b}$ & $1469 \mathrm{a}$ & $2091 \mathrm{a}$ & $1271 \mathrm{~b}$ & $-650 \mathrm{ab}$ & $622 \mathrm{a}$ & $71.98 \mathrm{a}$ \\
\hline & Suxiangjing NO.3 & $2460 \mathrm{e}$ & $1342 \mathrm{a}$ & $1939 a$ & $1118 \mathrm{~b}$ & $-521 \mathrm{a}$ & $598 \mathrm{ab}$ & $73.23 \mathrm{a}$ \\
\hline & Mean & 2627 & 1382 & 1977 & 1246 & -651 & 595 & 72.40 \\
\hline \multicolumn{8}{|l|}{2019} & $72.78 \mathrm{ab}$ \\
\hline \multirow{3}{*}{ HGY } & Nanjing9108 & 2549 b & $1606 \mathrm{ab}$ & $2214 \mathrm{ab}$ & $943 c$ & $-335 \mathrm{a}$ & $608 \mathrm{~b}$ & $71.75 \mathrm{bc}$ \\
\hline & Su1785 & $2644 \mathrm{a}$ & $1596 \mathrm{ab}$ & $2158 \mathrm{ab}$ & $1048 \mathrm{c}$ & $-486 a b$ & 562 c & $71.15 \mathrm{c}$ \\
\hline & Mean & 2790 & 1652 & 2187 & 1138 & -603 & 535 & 71.89 \\
\hline \multirow{4}{*}{ LGY } & Songzaoxiang NO.1 & $2528 \mathrm{~b}$ & $1285 \mathrm{~d}$ & $1891 \mathrm{c}$ & $1244 \mathrm{~b}$ & $-638 \mathrm{~b}$ & $606 \mathrm{~b}$ & $72.38 \mathrm{ab}$ \\
\hline & Changruan07-5 & 2546 b & 1492 bc & $2139 a b$ & $1054 \mathrm{c}$ & $-407 a$ & $647 \mathrm{ab}$ & $72.78 \mathrm{ab}$ \\
\hline & Suxiangjing NO.3 & $2389 \mathrm{c}$ & $1393 \mathrm{~cd}$ & $2063 \mathrm{~b}$ & $996 \mathrm{c}$ & $-326 a$ & $670 \mathrm{a}$ & $73.63 \mathrm{a}$ \\
\hline & Mean & 2488 & 1390 & 2031 & 1098 & -457 & 641 & 72.93 \\
\hline \multicolumn{9}{|c|}{ Analysis of variance } \\
\hline & Year $(\mathrm{Y})$ & $* *$ & $* *$ & $* *$ & $* *$ & $* *$ & $* *$ & ns \\
\hline & Type (T) & ** & $* *$ & $* *$ & $* *$ & * & $* *$ & $* *$ \\
\hline & Variety (V) & $* *$ & $* *$ & $* *$ & $* *$ & $* *$ & $* *$ & $* *$ \\
\hline & $\mathrm{Y} \times \mathrm{T}$ & $* *$ & $*$ & $*$ & ns & ns & ns & ns \\
\hline & $\mathrm{Y} \times \mathrm{V}$ & $*$ & ns & ns & ns & ns & ns & ns \\
\hline
\end{tabular}

HGY, high-yield varieties; LGY, low-yield varieties. Values within the same year followed by different letters are significantly different at the 0.05 probability level. * significant at the 0.05 probability level, ${ }^{* *}$ significant at the 0.01 probability level, ns not significant at the 0.05 probability level.

\subsubsection{Nutrition and Eating Quality}

There was no significant difference in amylose content between the HGY and LGY varieties (Table 8). The protein content of the HGY varieties was significantly lower than that of the LGY varieties across both years: the average protein content of the LGY varieties was $22.55 \%$ higher in 2018 than that of the HGY varieties and $24.28 \%$ higher in 2019 than that of the HGY varieties. There were significant differences in eating quality between the HGY and LGY varieties. The average values of appearance, viscosity, and degree of balance of the HGY varieties were significantly higher than those of the LGY varieties, while hardness showed the opposite trend. The taste value of the HGY varieties was significantly higher than that of the LGY varieties across both years. The average taste value of the HGY varieties was 75.67 in 2018 and 74.50 in 2019 , which was $10.92 \%$ and $11.48 \%$ higher than that of the LGY varieties.

Table 8. Nutrition and eating quality of semi-waxy japonica rice varieties with different grain yields.

\begin{tabular}{|c|c|c|c|c|c|c|c|c|}
\hline \multirow[b]{2}{*}{ Type } & \multirow{2}{*}{ Variety } & \multirow{2}{*}{$\begin{array}{c}\text { Amylose } \\
\text { Content (\%) }\end{array}$} & \multirow{2}{*}{$\begin{array}{c}\text { Protein } \\
\text { Content (\%) }\end{array}$} & \multicolumn{5}{|c|}{ Eating Quality } \\
\hline & & & & Appearance & Hardness & Viscosity & $\begin{array}{c}\text { Degree of } \\
\text { Balance }\end{array}$ & Taste Value \\
\hline \multicolumn{9}{|l|}{2018} \\
\hline \multirow{5}{*}{ HGY } & Nanjing5718 & $10.45 \mathrm{ab}$ & $7.12 \mathrm{c}$ & $6.37 \mathrm{~b}$ & $6.67 \mathrm{a}$ & $6.77 \mathrm{ab}$ & $6.37 \mathrm{~b}$ & $72.17 \mathrm{~b}$ \\
\hline & Nanjing9108 & $10.04 \mathrm{~b}$ & $6.20 \mathrm{~d}$ & $7.90 \mathrm{a}$ & $5.80 \mathrm{c}$ & $7.87 \mathrm{a}$ & $7.97 \mathrm{a}$ & $78.40 \mathrm{a}$ \\
\hline & Su1785 & $8.92 \mathrm{c}$ & $6.25 \mathrm{~d}$ & $7.47 \mathrm{a}$ & $6.23 \mathrm{~b}$ & $8.03 \mathrm{a}$ & $7.63 \mathrm{a}$ & $76.43 \mathrm{a}$ \\
\hline & Mean & 9.80 & 6.52 & 7.24 & 6.23 & 7.56 & 7.32 & 75.67 \\
\hline & Songzaoxiang NO.1 & $9.80 \mathrm{~b}$ & $8.28 \mathrm{a}$ & $6.53 \mathrm{~b}$ & $6.73 \mathrm{a}$ & $6.53 \mathrm{ab}$ & $6.60 \mathrm{~b}$ & $68.13 c$ \\
\hline \multirow{3}{*}{ LGY } & Changruan 07-5 & $10.85 \mathrm{a}$ & $7.61 \mathrm{~b}$ & $6.07 \mathrm{~b}$ & $6.60 \mathrm{a}$ & $6.50 \mathrm{ab}$ & $6.03 \mathrm{~b}$ & $69.37 \mathrm{c}$ \\
\hline & Suxiangiing NO.3 & $8.72 \mathrm{c}$ & $8.10 \mathrm{a}$ & $6.07 \mathrm{~b}$ & $6.77 \mathrm{a}$ & $6.10 \mathrm{~b}$ & $6.00 \mathrm{~b}$ & $67.17 \mathrm{c}$ \\
\hline & Mean & 9.79 & 7.99 & 6.22 & 6.70 & 6.38 & 6.21 & 68.22 \\
\hline \multirow{4}{*}{ HGY } & Nanjing5718 & $10.44 \mathrm{a}$ & $7.11 \mathrm{c}$ & $6.60 \mathrm{~b}$ & $6.65 \mathrm{a}$ & $7.65 \mathrm{abc}$ & $6.80 \mathrm{~b}$ & $70.50 \mathrm{~b}$ \\
\hline & Nanjing9108 & $10.08 \mathrm{ab}$ & $6.33 \mathrm{~d}$ & $7.40 \mathrm{a}$ & $6.25 \mathrm{a}$ & $7.95 \mathrm{ab}$ & $7.50 \mathrm{a}$ & $76.00 \mathrm{a}$ \\
\hline & Su1785 & $9.89 \mathrm{ab}$ & $6.46 \mathrm{~d}$ & $7.60 \mathrm{a}$ & $6.20 \mathrm{a}$ & $8.25 \mathrm{a}$ & $7.75 \mathrm{a}$ & $77.00 \mathrm{a}$ \\
\hline & Mean & 10.14 & $\begin{array}{c}0.40 \mathrm{u} \\
6.63\end{array}$ & $\begin{array}{l}7.00 \mathrm{~d} \\
7.20\end{array}$ & $\begin{array}{c}0.20 \mathrm{a} \\
6.37\end{array}$ & $\begin{array}{l}0.20 \mathrm{~d} \\
7.95\end{array}$ & $\begin{array}{l}7.35 \mathrm{a} \\
7.35\end{array}$ & 74.50 \\
\hline
\end{tabular}


Table 8. Cont.

\begin{tabular}{|c|c|c|c|c|c|c|c|c|}
\hline \multirow{2}{*}{ Type } & \multirow{2}{*}{ Variety } & \multirow{2}{*}{$\begin{array}{c}\text { Amylose } \\
\text { Content (\%) }\end{array}$} & \multirow{2}{*}{$\begin{array}{c}\text { Protein } \\
\text { Content (\%) }\end{array}$} & \multicolumn{5}{|c|}{ Eating Quality } \\
\hline & & & & Appearance & Hardness & Viscosity & $\begin{array}{c}\text { Degree of } \\
\text { Balance }\end{array}$ & Taste Value \\
\hline \multirow{4}{*}{ LGY } & Songzaoxiang NO.1 & $9.75 \mathrm{ab}$ & $8.53 \mathrm{a}$ & $6.15 \mathrm{~b}$ & $7.15 \mathrm{a}$ & $6.85 \mathrm{~cd}$ & $6.35 \mathrm{bc}$ & $66.50 c$ \\
\hline & Changruan07-5 & $10.59 \mathrm{a}$ & $7.93 \mathrm{~b}$ & $7.45 \mathrm{a}$ & $6.85 \mathrm{a}$ & $7.15 \mathrm{bcd}$ & $7.60 \mathrm{a}$ & $68.50 \mathrm{c}$ \\
\hline & Suxiangijing NO.3 & $9.34 \mathrm{~b}$ & $8.26 \mathrm{a}$ & $5.95 \mathrm{~b}$ & $7.00 \mathrm{a}$ & $6.25 \mathrm{~d}$ & $5.90 \mathrm{c}$ & $65.50 c$ \\
\hline & Mean & 9.90 & 8.24 & 6.52 & 7.00 & 6.75 & 6.62 & 66.83 \\
\hline \multicolumn{9}{|c|}{ Analysis of variance } \\
\hline & Year $(Y)$ & ns & $* *$ & ns & ns & * & ns & $*$ \\
\hline & Type (T) & ns & $* *$ & $* *$ & $* *$ & $* *$ & $* *$ & ** \\
\hline & Variety $(\mathrm{V})$ & $* *$ & $* *$ & $* *$ & ns & $*$ & $* *$ & ** \\
\hline & $\mathrm{Y} \times \mathrm{T}$ & ns & ns & ns & ns & ns & ns & ns \\
\hline & $\mathrm{Y} \times \mathrm{V}$ & $*$ & ns & $* *$ & ns & ns & $* *$ & ns \\
\hline
\end{tabular}

HGY, high-yield varieties; LGY, low-yield varieties. Values within the same year followed by different letters are significantly different at the 0.05 probability level. * significant at the 0.05 probability level, ** significant at the 0.01 probability level, ns not significant at the 0.05 probability level.

\section{Discussion}

\subsection{Grain Yield Formation Characteristics of Semi-Waxy japonica Rice with a High Grain Yield}

Good plant type traits, high dry matter accumulation, and sufficient total spikelet number were the important bases for achieving high rice grain yield [20-23]. Wu et al. [24] showed that to obtain a rice grain yield above $11.7 \mathrm{t} \mathrm{hm}^{-2}$, the total spikelet number per square meter should be more than 45,000 , the filled grain rate should be above $90 \%$, and the 1000 -grain weight should be above $26 \mathrm{~g}$. In this study, the average total spikelet number of the HGY varieties was $18.42 \%$ higher in 2018 and $22.28 \%$ higher in 2019 than that of the LGY varieties, which resulted in the average grain yield of the HGY varieties being 29.39 and 30.79\% higher than those of the LGY varieties in 2018 and 2019, respectively (Table 2). The total spikelet number was mainly determined by the effective panicle number and spikelet per panicle. Compared with the LGY varieties, the HGY varieties had a smaller effective panicle number per area, but more spikelet per panicle (Table 2), which indicated that more spikelet per panicle was the main reason for the observed higher total spikelet number of the HGY varieties [25,26]. In addition, we noted that the increase in the ratio of grain yield was greater than that of the total spikelet number, which may be related to the higher 1000-grain weight of the HGY varieties (Table 2). Therefore, we speculated that more spikelet per panicle and a larger 1000-grain weight are primary approaches for achieving a high yield with semi-waxy japonica rice, which is consistent with previous research [27].

To fill the huge sink formed by a great number of spikelets, the morphological and function of leaves, stems, and roots of high-yielding rice need to be better than those of lowyield rice. For the high-yield rice varieties, the leaf area index and the percentages of the highly effective leaf area were comparatively high [9], the decreasing rate of leaf area was low [28], and the top three leaves were upright and straight, with a lower leaf angle [29,30]. In addition, the longer the total growth period, especially from elongating to maturity, allows the amassing of more temperature and radiation resources, which is conducive to increased dry matter accumulation [5,9]. Our research also showed similar results: the leaf area indexes of the HGY varieties were significantly higher than those of the LGY varieties at the elongating, heading, and maturity stages in both years (Table 4). After heading, due to the larger length and width of the top three leaves of the HGY varieties (Table 5), the high effective leaf area and the ratio of the HGY varieties were significantly higher than those of the LGY varieties, and the average decreasing rate of the leaf area of the HGY varieties was lower than that of the LGY varieties (Table 4). The HGY varieties had a longer total growth duration, especially from elongating to maturity (Table 1 ). Moreover, the leaf angle of the top 3 leaves in the HGY varieties was smaller (Table 5), which was beneficial to increasing light energy capture and forming a high light efficiency population [31]. We hypothesized that the good morphology and function of leaves and stems, and longer growth duration, resulted in a high above-ground biomass accumulation of the HGY varieties. However, rice grain yield can also be expressed as the product of biomass accumulation and harvest index. Since there was no significant difference in harvest index between the HGY and 
LGY varieties, we speculated that the difference in above-ground biomass accumulation was another major reason for the difference in rice yield, which was consistent with the results of previous studies [23,32].

The high-yield rice varieties with more grains per panicle generally have a low filled grain rate [33,34]. In this study, we also found that the average filled grain rate of the HGY varieties was significantly lower than that of the LGY varieties (Table 2), indicating that the assimilates of the high-yield varieties were not sufficient [35]. It should be noted that Nanjing5718 (a high-yield variety) not only had the highest grain yield, but also had an average filled grain rate of $97.68 \%$, which did not differ significantly from that of the LGY varieties (Table 2). We speculated that the reasons for Nanjing5718 having a higher filled grain rate might be related to it having the highest single stem-sheath weight (Table 5). A Higher single stem-sheath weight can improve the filled grain rate because the assimilate accumulated in the stem-sheath at the heading stage can be translocated to support grain filling $[34,36]$. These findings indicate that the grain yield of the HGY varieties could be further improved by cultivating higher single stem-sheath weight.

\subsection{Rice Quality Characteristics of Semi-Waxy japonica Rice with a High Grain Yield}

Next to grain yield, rice quality is the most important factor in rice production as it is directly related to market value and thus influences farmer incomes. Rice quality is a complex and comprehensive metric, including milling quality, appearance quality, cooking and eating quality, etc.

The milling quality is an important index affecting the economic value of rice, including brown rice rate, milled rice rate, and head milled rice rate [37]. Previous studies showed a positive correlation between the milled rice rate and yield, but there was no significant correlation between the head milled rice rate and yield [38]. In this study, we found no significant difference in the brown rice rate and milled rice rate across different grain yield types, but the head milled rice rate of the HGY varieties was significantly lower than that of the LGY varieties (Table 6). The head milled rice rate is influenced by grain characteristics, such as chalkiness, grain shape, and grain moisture [39]. Previous studies have shown that larger grains are more likely trend to break during milling [40]. In this study, the 1000-grain weight of the HGY varieties was significantly higher than that of the LGY varieties (Table 2), indicating that the grain size of the HGY varieties was significantly larger than that of the LGY varieties, and it was easier to be broken during milling. Leesawatwong et al. [41] and Balindong et al. [42] reported that increasing the grain protein content can enhance the hardness of grains and reduce grain breakage during milling, thus increasing the head rice rate. Generally, protein content in rice grains is negatively correlated with grain yield and grain weight [43]. Our results also showed that the grain protein content of the HGY varieties was significantly lower than that of the LGY varieties (Table 8), which may be one of the reasons for the low head milled rice rate of the HGY varieties.

Chalkiness not only affects appearance quality but also milling quality, so it is a key determinant of the commercial value of milled rice. It has been well documented that chalkiness is controlled by polygenes, resulting from the loose packing of starch granules in the grain endosperm [44,45]. Cai et al. [38] suggested that ratoon rice with low grain yield had lower chalkiness than the main crop rice with a high grain yield. In this study, we also found that the average chalky kernel rate, chalky area, and chalkiness degree of the HGY varieties were significantly higher than those of the LGY varieties (Table 6). One reason for this result might be that the HGY varieties had a higher 1000-grain weight (Table 2), which tends to result in loose starch granules and higher chalkiness $[15,46]$. The high degree of chalkiness indicates that rice grains have lower starch density and are more easily broken during milling, so chalkiness is generally negatively correlated with processing quality [47]. This may be one of the reasons for the low head milled rice rate in HGY varieties.

Eating quality is the most important aspect of evaluating rice quality. The cooked rice texture, including hardness and stickiness, are important determinants of eating quality [48]. Previous studies have shown that the hardness and stickiness of cooked rice are closely 
related to amylose and protein contents, which can limit starch swelling and leaching during cooking [49-51]. Rice higher in amylose or protein has a harder, less-sticky texture after cooking, representing low eating quality [51,52]. Consistent with previous studies [12,52], there was no significant difference in amylose content between the HGY and LGY varieties (Table 8) in this study, indicating that there was no trade-off between amylose content and grain yield. Compared with the LGY varieties, the protein content of the HGY varieties was significantly lower (Table 8), which may be primarily due to the larger sink capacity of HGY varieties, which can dilute the grain protein content [53]. The results of our previous study showed that protein content was the main factor affecting the eating quality of semi-waxy japonica rice varieties [54]. Therefore, we speculated that the HGY varieties would have a higher appearance, viscosity, degree of balance, taste value, and lower hardness (Table 8) related to their lower grain protein content. In addition, the rice pasting properties revealed by RVA measurements reflected the swelling ability of starch granules, which predicts the texture of cooked rice [55]. Among the pasting property parameters, breakdown and setback values are considered important indexes in the evaluation of eating quality [56]. Generally, rice with good eating quality has a higher breakdown value and lower setback value [57]. In this study, we observed that the HGY varieties had a higher breakdown value and lower setback value (Table 7), which indicated that the HGY varieties had a better eating quality.

\section{Conclusions}

The grain yield superiority of the HGY varieties was mainly attributable to more spikelet per panicle and a larger 1000-grain weight. The underlying factors were a larger leaf area index at each growth stage, larger high efficient leaf area formed by the increased length and width of the top three leaves, smaller leaf angles of the top three leaves, greater single stem-sheath weight, more total dry matter accumulation, and longer growth duration from elongation to maturity. Due to the low protein content in grain, the HGY varieties had a better eating quality than the LGY varieties, and due to the low protein content and the high 1000-grain weight, the head milled rice rate and appearance quality of the HGY varieties were inferior to the LGY varieties. In conclusion, choosing the semiwaxy japonica varieties with more spikelet per panicle and higher 1000-grain weight can achieve the synergistic improvement of grain yield and eating quality, but how to improve the milling quality and appearance quality of high-yield semi-waxy japonica rice needs further research.

Author Contributions: Funding acquisition, H.W. and H.Z.; investigation, Q.L., Y.T., S.C., L.Z., and J.T.; Project administration, H.W. and H.Z.; writing—original draft, Q.L.; writing—review and editing, Z.X. and H.W. All authors have read and agreed to the published version of the manuscript.

Funding: This research was funded by the National Key Research Program of China, grant number 2016YFD0300503; the Key Research Program of Jiangsu Province, China, grant numbers BE2016344 and BE2018355; the Project Funded by the Priority Academic Program Development of Jiangsu Higher Education Institutions, China; the Open Program of Jiangsu Key Laboratory of Crop Genetics and Physiology, China, grant number YCSL201907; and the National Natural Science Foundation of China, grant number 31801293.

Institutional Review Board Statement: Not applicable.

Informed Consent Statement: Not applicable.

Conflicts of Interest: The authors declare no conflict of interest.

\section{References}

1. Ray, D.K.; Mueller, N.D.; West, P.C.; Foley, J.A.; Hart, J.P. Yield trends are insufficient to double global crop production by 2050. PLOS ONE 2013, 8, e66428. [CrossRef]

2. Virmani, S.S.; Edwards, I.B. Current status and future prospects for breeding hybrid rice and wheat. Adv. Agron. 1983, 36, 145-214. [CrossRef]

3. Yuan, L.P. Hybrid rice breeding for super high yield. Hybrid Rice 1997, 12, 1-3. [CrossRef] 
4. Lee, J.W.; Hong, K. Economic growth in Asia: Determinants and prospects. Jpn. World Econ. 2010, 24, 101-113. [CrossRef]

5. Wei, H.H.; Meng, T.Y.; Li, C.; Xu, K.; Huo, Z.Y.; Wei, H.Y.; Guo, B.W.; Zhang, H.C.; Dai, Q.G. Comparisons of grain yield and nutrient accumulation and translocation in high-yielding japonica/indica hybrids, indica hybrids, and japonica conventional varieties. Field Crops Res. 2017, 204, 101-109. [CrossRef]

6. Jiang, Y.H.; Zhang, H.C.; Zhao, K.; Xu, J.W.; Wei, H.H.; Long, H.Y.; Wang, W.T.; Dai, Q.G.; Huo, Z.Y.; Xu, K. Difference in yield and its components characteristics of different type rice cultivars in the lower reaches of the Yangtze River. Chin. J. Rice Sci. 2014, 28, 621-631. [CrossRef]

7. Katsura, K.; Maeda, S.; Horie, T.; Shiraiwa, T. Analysis of yield attributes and crop physiological traits of Liangyoupeijiu, a hybrid rice recently bred in China. Field Crops Res. 2007, 103, 170-177. [CrossRef]

8. Wei, H.Y.; Hu, L.; Zhu, Y.; Xu, D.; Zheng, L.M.; Chen, Z.F.; Hu, Y.J.; Cui, P.Y.; Guo, B.W.; Dai, Q.G.; et al. Different characteristics of nutrient absorption and utilization between inbred japonica super rice and inter-sub-specific hybrid super rice. Field Crops Res. 2018, 218, 88-96. [CrossRef]

9. Wei, H.Y.; Zhang, H.C.; Blumwald, E.; Li, H.L.; Cheng, J.Q.; Dai, Q.G.; Huo, Z.Y.; Xu, K.; Guo, B.W. Different characteristics of high yield formation between inbred japonica super rice and inter-sub-specific hybrid super rice. Field Crops Res. 2016, 198, 179-187. [CrossRef]

10. Peng, S.B.; Tang, Q.Y.; Zou, Y.B. Current status and challenges of rice production in China. Plant Prod. Sci. 2009, 12, 3-8. [CrossRef]

11. Zhu, D.W.; Zhang, H.C.; Guo, B.W.; Xu, K.; Dai, Q.G.; Wei, H.Y.; Gao, H.; Hu, Y.J.; Cui, P.Y.; Huo, Z.Y. Effects of nitro-gen level on yield and quality of japonica soft super rice. J. Integr. Agric. 2017, 16, 1018-1027. [CrossRef]

12. Hu, L.; Zhu, Y.; Xu, D.; Chen, Z.F.; Hu, B.Q.; Han, C.; Qiu, S.; Wu, P.; Zhang, H.C.; Wei, H.Y. Characteristics of good taste and high yield type of single cropping late japonica rice in southern china. Sci. Agric. Sin. 2019, 52, 215-227. [CrossRef]

13. Fahad, S.; Hussain, S.; Saud, S.; Hassan, S.; Chauhan, B.S.; Khan, F.; Ihsan, M.Z.; Ullah, A.; Wu, C.; Bajwa, A.A.; et al. Responses of rapid viscoanalyzer profile and other rice grain qualities to exogenously applied plant growth regulators under high day and high night temperatures. PLoS ONE 2016, 11, e0159590. [CrossRef]

14. Fahad, S.; Hussain, S.; Saud, S.; Hassan, S.; Tanveer, M.; Ihsan, M.Z.; Shah, A.N.; Ullah, A.; Nasrullah, K.F.; Ullah, S.; et al. A combined application of biochar and phosphorus alleviates heat-induced adversities on physiological, agronomical and quality attributes of rice. Plant Physiol. Biochem. 2016, 103, 191-198. [CrossRef]

15. Xu, Q.; Chen, W.F.; Xu, Z.J. Relationship between grain yield and quality in rice germplasms grown across different growing areas. Breed. Sci. 2015, 65, 226-232. [CrossRef]

16. Gu, J.F.; Chen, J.; Chen, L.; Wang, Z.Q.; Zhang, H.; Yang, J.C. Grain quality changes and responses to nitrogen fertilizer of japonica rice cultivars released in the Yangtze River Basin from the 1950s to 2000s. Crop J. 2015, 3, 285-297. [CrossRef]

17. Yue, H.L.; Zhao, Q.Y.; Zhao, C.F.; Tian, Z.; Chen, T.; Liang, W.H.; Zhang, Y.D.; Wang, Z.H.; Wang, C.L. Characteristics of edible quality and their relationship with sensory evaluation of semi-waxy japonica rice varieties from Jiangsu Province. J. Chin. Cereals Oils Assoc. 2020, 35, 7-14. [CrossRef]

18. Zhao, C.F.; Yue, H.L.; Huang, S.J.; Zhou, L.H.; Zhao, L.; Zhang, Y.D.; Chen, T.; Zhu, Z.; Zhao, Q.Y.; Yao, S.; et al. Eating quality and physicochemical properties in Nanjing rice varieties. Sci. Agric. Sin. 2019, 52, 909-920. [CrossRef]

19. National Standards of P.R.C. (GB/T17891-2017); "High Quality Paddy". Standards Press of China: Beijing, China, 2017.

20. Gravois, K.A.; Helms, R.S. Path analysis of rice yield and yield components as affected by seeding rate. Agron. J. 1992, 84, 1-4. [CrossRef]

21. Sheehy, J.; Dionora, M.; Mitchell, P. Spikelet numbers: Sink size and potential yield in rice. Field Crops Res. 2001, 71, 77-85. [CrossRef]

22. Zhang, H.; Xue, Y.; Wang, Z.Q.; Yang, J.C.; Zhang, J.H. Morphological and physiological traits of roots and their relationships with shoot growth in super rice. Field Crops Res. 2009, 113, 31-40. [CrossRef]

23. Ao, H.J.; Wang, S.H.; Zou, Y.B.; Peng, S.B.; Tang, Q.Y.; Fang, Y.X.; Xiao, A.M.; Chen, Y.M.; Xiong, C.M. Study on yield stability and dry matter characteristics of super hybrid rice. Sci. Agric. Sin. 2008, 41, 1927-1936. [CrossRef]

24. Wu, G.C.; Zhang, H.C.; Qian, Y.F.; Li, D.J.; Zhou, Y.Y.; Xu, J.; Wu, W.G.; Dai, Q.G.; Huo, Z.Y.; Xu, K. Rule of grain yield components from high yield to super high yield and the characters of super-high yielding Japonica super rice. Sci. Agric. Sin. 2010, 43, 266-276. [CrossRef]

25. Wu, G.C.; Zhang, H.C.; Dai, Q.G.; Huo, Z.Y.; Xu, K.; Gao, H.; Wei, H.Y.; Sha, A.Q.; Xu, Z.J.; Qian, Z.H.; et al. Characteristics of dry matter production and accumulation and super-high yield of japonica super rice in South China. Acta Agron. Sin. 2010, 36, 1921-1930. [CrossRef]

26. Yang, J.C.; Du, Y.; Wu, C.F.; Liu, L.J.; Wang, Z.Q.; Zhu, Q.S. Growth and development characteristics of super-high-yielding mid-season japonica rice. Sci. Agric. Sin. 2006, 39, 1336-1345. [CrossRef]

27. Gong, J.L.; Hu, Y.J.; Long, H.Y.; Chang, Y.; Li, J.; Zhang, H.C.; Ma, R.R.; Wang, X.Y.; Dai, Q.G.; Huo, Z.Y.; et al. Study on collaborating characteristics of grain yield components and panicle traits of large panicle hybrid japonica rice. Sci. Agric. Sin. 2012, 45, 2147-2158. [CrossRef]

28. Lee, R.H.; Wang, C.H.; Huang, L.T.; Chen, S.C.G. Leaf senescence in rice plants: Cloning and characterization of senescence up-regulated genes. J. Exp. Bot. 2001, 52, 1117-1121. [CrossRef] 
29. Gong, J.L.; Xing, Z.P.; Hu, Y.J.; Zhang, H.C.; Dai, Q.G.; Huo, Z.Y.; Xu, K.; Wei, H.Y.; Gao, H. Difference of characteristics of photosynthesis: Matter production and translocation between indica and japonica super rice. Acta Agron. Sin. 2014, 40, 497-510. [CrossRef]

30. Wei, H.H.; Yang, Y.L.; Shao, X.Y.; Shi, T.Y.; Meng, T.Y.; Lu, Y.; Tao, Y.; Li, X.Y.; Ding, E.H.; Chen, Y.L.; et al. Higher leaf area through leaf width and lower leaf angle were the primary morphological traits for yield advantage of japonica/indica hybrids. J. Integr. Agric. 2020, 19, 483-494. [CrossRef]

31. Ling, Q.H.; Zhang, H.C.; Su, Z.F.; Guo, W.S.; Chen, D.H.; Lu, W.P.; Leng, S.H.; Ling, L.; Yang, J.C.; Ding, Y.F.; et al. Quality of Crop Population; Shanghai Scientific and Technical Publishers: Shanghai, China, 2000; pp. 44-85.

32. Wu, W.G.; Zhang, H.C.; Qian, Y.F.; Cheng, Y.; Wu, G.C.; Zhai, C.Q.; Dai, Q.G. Analysis on Dry Matter Production Characteristics of Super Hybrid Rice. Rice Sci. 2008, 15, 110-118. [CrossRef]

33. Kato, T.; Shinmura, D.; Taniguchi, A. Activities of enzymes for sucrose-starch conversion in developing endosperm of rice and their association with grain filling in extra-heavy panicle types. Plant Prod. Sci. 2007, 10, 442-450. [CrossRef]

34. Chang, S.Q.; Chang, T.G.; Song, Q.F.; Zhu, X.G.; Deng, Q.Y. Photosynthetic and agronomic traits of an elite hybrid rice Y-Liang-You 900 with a record-high yield. Field Crops Res. 2016, 187, 49-57. [CrossRef]

35. Li, J.; Xiang, X.; He, L.; Li, P. Source-sink relationship in inter-sub-specific hybrid rice. Rice Sci. 2006, 13, $250-256$.

36. Katsura, K.; Maeda, S.; Lubis, I.; Horie, T.; Cao, W.; Shiraiwa, T. The high yield of irrigated rice in Yunnan, China-'A cross-location analysis'. Field Crops Res. 2008, 107, 1-11. [CrossRef]

37. Mao, T.; Li, X.; Jiang, S.K.; Tang, L.; Wang, J.Y.; Xu, H.; Xu, Z.J. Discussion on strategy of grain quality improvement for super high yielding japonica rice in Northeast China. J. Integr. Agric. 2017, 16, 1075-1083. [CrossRef]

38. Cai, H.Y.; Tabien, R.E.; Xu, D.; Harper, C.L.; Samford, J.; Yang, Y.Y.; You, A.Q.; Samonte, S.O.; Holgate, L.; Jiao, C.H. Grain quality and yield of rice in the main and ratoon harvests in the southern U.S. J. Agric. Sci. 2019, 11, 1-13. [CrossRef]

39. Moldenhauer, K.A.K.; Gibbons, J.H.; McKenzie, K.S. Rice Varieties. Rice: Chemistry and Technology; Champagne, E.T., Ed.; The American Association of Cereal Chemists: St. Paul, MN, USA, 2004; pp. 49-75.

40. Nelson, J.C.; Mcclung, A.M.; Fjellstrom, R.G.; Moldenhauer, K.A.K.; Boza, E.; Jodari, F.; Yeater, K.M. Mapping QTL main and interaction influences on milling quality in elite US rice germplasm. Theor. Appl. Genet. 2011, 122, 291-309. [CrossRef]

41. Leesawatwong, M.; Jamjod, S.; Kuo, J.; Dell, B.; Rerkasem, B. Nitrogen fertilizer increases seed protein and milling quality of rice. Cereal Chem. 2005, 82, 588-593. [CrossRef]

42. Balindong, J.L.; Ward, R.M.; Rose, T.J.; Liu, L.; Raymond, C.A.; Snell, P.J.; Ovenden, B.W.; Waters, D.L.E. Rice grain protein composition influences head rice yield. Cereal Chem. 2018, 95, 1-11. [CrossRef]

43. Yu, Y.H.; Li, G.; Fan, Y.Y.; Zhang, K.Q.; Min, J.; Zhu, Z.W.; Zhuang, J.Y. Genetic relationship between grain yield and contents of protein and fat in a recombinant inbred population of rice. J. Cereal Sci. 2009, 50, 121-125. [CrossRef]

44. Gong, J.Y.; Miao, J.H.; Zhao, Y.; Zhao, Q.; Feng, Q.; Zhan, Q.L.; Han, B. Dissecting genetic basis of grain shape and chalkiness traits in hybrid rice using multiple collaborative populations. Mol. Plant 2017, 10, 1353-1356. [CrossRef] [PubMed]

45. Lu, Y.; Zhang, X.M.; Qi, Y.; Zhang, C.Q.; Ling, Y.P.; Liu, Q.Q. Scanning electron microscopic analysis of grain cross-section from rice with different transparency. Chin. J. Rice Sci. 2018, 32, 189-199. [CrossRef]

46. Song, X.J.; Huang, W.; Shi, M.; Zhu, M.Z.; Lin, H.X. A QTL for rice grain width and weight encodes a previously unknown RING-type E3 ubiquitin ligase. Nat. Genet. 2007, 39, 623-630. [CrossRef] [PubMed]

47. Yang, L.X.; Wang, Y.L.; Dong, G.C.; Gu, H.; Huang, J.Y.; Zhu, J.G.; Yang, H.L.; Liu, G.; Han, Y. The impact of free-air CO2 enrichment (FACE) and nitrogen supply on grain quality of rice. Field Crop Res. 2007, 102, 128-140. [CrossRef]

48. Suwannaporn, P.; Linnemann, A. Rice-eating quality among consumers in different rice grain preference countries. J. Sens. Stud. 2008, 23, 1-13. [CrossRef]

49. Martin, M.; Fitzgerald, M.A. Proteins in rice grains influence cooking properties. J. Cereal Sci. 2002, 36, 285-294. [CrossRef]

50. Derycke, V.; Veraverbeke, W.S.; Vandeputte, G.E.; Deman, W.; Hoseney, C.; Delcour, J.A. Impact of proteins on pasting and cooking properties of non-parboiled and parboiled rice. Cereal Chem. 2005, 82, 468-474. [CrossRef]

51. Li, H.Y.; Prakash, S.; Nicholson, T.M.; Fitzgerald, M.A.; Gilbert, R.G. The importance of amylose and amylopectin fine structure for textural properties of cooked rice grains. Food Chem. 2016, 196, 702-711. [CrossRef]

52. Bian, J.L.; Ren, G.L.; Han, C.; Xu, F.F.; Qiu, S.; Tang, J.H.; Zhang, H.C.; Wei, H.Y.; Gao, H. Comparative analysis on grain quality and yield of different panicle weight indica-japonica hybrid rice (Oryza sativa L.) cultivars. J. Integr. Agric. 2020, 19, 999-1009. [CrossRef]

53. Tadashi, T.; Sumiyo, N.; Yuri, M. Cultivar differences in the grain protein accumulation ability in rice (Oryza sativa L.). Field Crops Res. 2016, 192, 110-117. [CrossRef]

54. Liu, Q.Y.; Tao, Y.; Cheng, S.; Zhou, L.; Tian, J.Y.; Xing, Z.P.; Liu, G.D.; Wei, H.Y.; Zhang, H.C. Relating amylose and protein contents to eating quality in 105 varieties of Japonica rice. Cereal Chem. 2020, 97, 1303-1312. [CrossRef]

55. Wani, A.A.; Singh, P.; Shah, M.A.; Schweiggert-Weisz, U.; Gul, K.; Wani, I.A. Rice starch diversity: Effects on structural, morphological, thermal, and physicochemical properties-A review. Compr. Rev. Food Sci. Food Saf. 2012, 11, 417-436. [CrossRef]

56. Zhu, D.W.; Zhang, H.C.; Guo, B.W.; Xu, K.; Dai, Q.G.; Wei, C.X.; Zhou, G.S.; Huo, Z.Y. Physicochemical properties of indicajaponica hybrid rice starch from Chinese varieties. Food Hydrocoll. 2017, 63, 356-363. [CrossRef]

57. Asante, M.D.; Offei, S.K.; Grance, V.; Adu-Dapaah, H.; Danquah, E.Y.; Bryant, R.; McClung, A. Starch physicochemical properties of rice accessions and their association with molecular markers. Starch 2013, 65, 1022-1028. [CrossRef] 